\title{
Retinal Hemorrhage after Epiduroscopy in a Patient using Dexa- METHASONE: A CASE REPORT
}

Heleen A. Biersteker, MD ${ }^{1,2}$, José W. Geurts, $\mathbf{M S c}^{3}$, and JanWillem Kallewaard, MD ${ }^{3}$

Epiduroscopy is a minimally invasive procedure used to diagnose epidural fibrosis and to release this fibrosis; epiduroscopy is also used to precisely deposit medication into the epidural space. It is commonly used in patients who are diagnosed with failed back surgery syndrome (FBSS) after more conservative treatment has failed to provide sufficient relief of symptoms.

A rare complication of epiduroscopy is retinal hemorrhaging, which is likely caused by overpressurization of the epidural space during the procedure. Patient-related risk factors for developing retinal hemorrhage after epiduroscopy remain largely unknown. This is the first case report of retinal hemorrhage in a patient using chronic dexamethasone.

We describe a 73-year-old man diagnosed with FBSS who underwent epiduroscopy to diagnose and relieve epidural fibrosis. The procedure was uneventful and he was discharged from our clinic the same day, but upon routine check-up he mentioned blurry vision. Immediately he was referred to an ophthalmologist who diagnosed retinal hemorrhages in both eyes upon fundoscopic examination. Our patient was using dexamethasone for the treatment of allergies. Three months after the procedure, his vision was restored fully in the right eye and $95 \%$ in the left eye.

Chronic corticosteroid use may weaken retinal veins, making them prone to rupture when there is increased pressure, even for a short period of time. Chronic use of corticosteroids must be considered a risk factor for developing retinal hemorrhages in patients undergoing epiduroscopy. Long-term use of corticosteroid can be considered as a relative contraindication for epiduroscopy.

Key words: Epiduroscopy, complications, interventional pain, corticosteroids, retinal hemmorhage, failed back surgery syndrome
Epiduroscopy is a minimally invasive diagnostic and therapeutic procedure in which the spinal epidural space is visualized by using a small steerable endoscope. Apart from visualizing normal and pathological

From: 'Department of Anesthesiology, Pain and Palliative Care, Radboud University Medical Center, Nijmegen, Gelderland, The Netherlands; ${ }^{2}$ Department of Anesthesiology and Pain Medicine, Catharina Hospital, Eindhoven, Noord-Brabant, The Netherlands; ${ }^{3}$ Department of Anesthesiology and Pain Medicine, Rijnstate, Arnhem, Gelderland, The Netherlands

Author for correspondence: JanWillem Kallewaard, MD

Address: Department of Anesthesiology and Pain Medicine, Rijnstate, Postbus 9555, 6800 TA, Arnhem, Gelderland, The Netherlands

E-mail: jkallewaard@rijnstate.nl structures in the epidural space, it allows for interventions such as adhesiolysis and precise deposition of steroids $(1,2)$.

Epiduroscopy is used in lumbosacral radicular pain, especially after back surgery. It is used when pain fails to respond to conservative and minor invasive therapies and before spinal cord stimulation is considered (3).

Risks of epiduroscopy are intravascular injection, intrathecal injection of contrast dye, dural puncture, epidural bleeding, and retinal hemorrhage (2).

Retinal hemorrhage following epiduroscopy or epidural injections is a rare but significant complication. 
Retinal hemorrhage after epidural injection was first reported in 1961 (4).

Retinal hemorrhages are probably caused by an increase in intracranial pressure (ICP); sudden distension of the epidural space compresses the subarachnoidal space with a rise in cerebrospinal fluid (CSF) pressure (5). The optic nerve is surrounded by an extension of the subarachnoid space; the central vein of the retina crosses this space to join the orbital veins. Retinal blood circulation depends on the pressure in the central vein being above CSF pressure. When the ICP rises above the pressure in the central vein, the pressure in the retinal veins rises and bleeding occurs.

Several precautions should be taken to minimize the risk of retinal bleeding during epiduroscopy. A slow injection rate of $1 \mathrm{~mL}$ per second and limitation of the amount of fluid injected is advised (4). During epiduroscopy, conscious sedation should be used; the patient should be able to report head and neck pain, which are indicators of increased epidural pressure. However, patient-related risk factors for developing retinal hemorrhage remain largely unknown since only a few cases have been reported in the medical literature (5).

A case of retinal hemorrhage after epiduroscopy is presented here in a patient who was using dexamethasone to treat his allergies.

\section{CASE REPORT}

Permission to report this case was obtained from the patient. A 73-year-old man was referred to our pain clinic by his general practitioner. His chief complaint was pain in his left leg and numbness of the left foot. Less than a year ago, he had undergone a left-sided laminectomy at the L4-5 level because of a herniated nucleus pulposus (HNP). Over the following weeks, his symptoms markedly improved. Unfortunately, his symptoms gradually returned after 2 weeks, for which he was treated with epidural injections of local anesthetics and corticosteroids and pulsed radiofrequency of the dorsal root ganglion at L5. Because this provided insufficient relief of his symptoms, a reoperation was performed 4 months after his initial surgery; during this operation a lot of epidural fibrosis was removed.
When he presented himself to our pain clinic, the pain in his leg followed the L5 dermatome; the pain was stabbing in nature and provoked by movement.

He was taking $50 \mathrm{mg}$ of diclofenac 3 times daily to alleviate his pain and $0.5 \mathrm{mg}$ of oral dexamethasone daily because of allergies.

Several types of opiates and antineuropathic medicine were prescribed previously, all with insufficient results or unacceptable side effects.

He was diagnosed with failed back surgery syndrome (FBSS) and after receiving extensive information and obtaining informed consent, he was scheduled to have an epiduroscopy.

When medication, conservative care, and minimal invasive pain treatments fail, epiduroscopy and spinal cord stimulation are possible treatment options for FBSS. Epiduroscopy is diagnostic by allowing visualization of fibroses and therapeutic when performing adhesiolysis, via the flushing of inflammatory mediators and deposition of targeted medication (6).

\section{Procedure}

After completion of the preoperative time-out procedure, $2 \mathrm{~g}$ of cefazoline were given intravenously and the patient was placed in the prone position. After disinfection and sterile draping, a steerable 3.0-mm Resascope (AMS Group, Italy) was inserted through the sacral hiatus into the epidural space. Contrast dye was injected and the epidurogram showed a stop at the left L5 level. The endoscope was slowly advanced towards this level with low-pressure flushing with 80 $\mathrm{mL}$ of normal saline. During the procedure, the pressure in the epidural space was monitored and stayed below the mean arterial blood pressure. Visibility of pulsations of the dura served as an extra way to check epidural pressure. Adhesions were seen at the L4-5 level and adhesiolysis was performed successfully with the opening of the filling defect and the spreading of contrast dye around the L5 nerve on the left side. Six $\mathrm{mg}$ of ropivacaine, $80 \mathrm{mg}$ of methylprednisolone, and $600 \mathrm{IE}$ of hyaluronidase were deposited after confirming with contrast dye that the medications stayed in the epidural space. During the procedure, the patient was sedated with a combination of midazolam, remifentanil, and propofol. Communication was possible throughout the procedure; the patient 
did not complain of head and neck pain. The total procedure time was 45 minutes. After an uneventful stay in the recovery room, the patient was discharged home the same day.

On routine follow-up a week after the procedure, the patient reported major improvement of his leg pain but also mentioned blurry vision. Symptoms started some days after the epiduroscopy. He was immediately referred to an ophthalmologist who saw multiple retinal hemorrhages in both eyes on fundoscopic examination (Fig. 1). The hemorrhages were mostly in the peripheral regions of the retina and not near the optical nerve. During the first couple of weeks, his vision deteriorated even more because of resorption edema surrounding the hemorrhages.

Three months after the procedure, the patient's leg pain was still fully gone; his vision was completely restored in the right eye and $95 \%$ restored in the left eye, with expectation of full recovery.

\section{DICUSSION}

Epiduroscopy is both a diagnostic and therapeutic intervention in patients with FBSS and associated with a low risk of complications $(1,2,7)$.

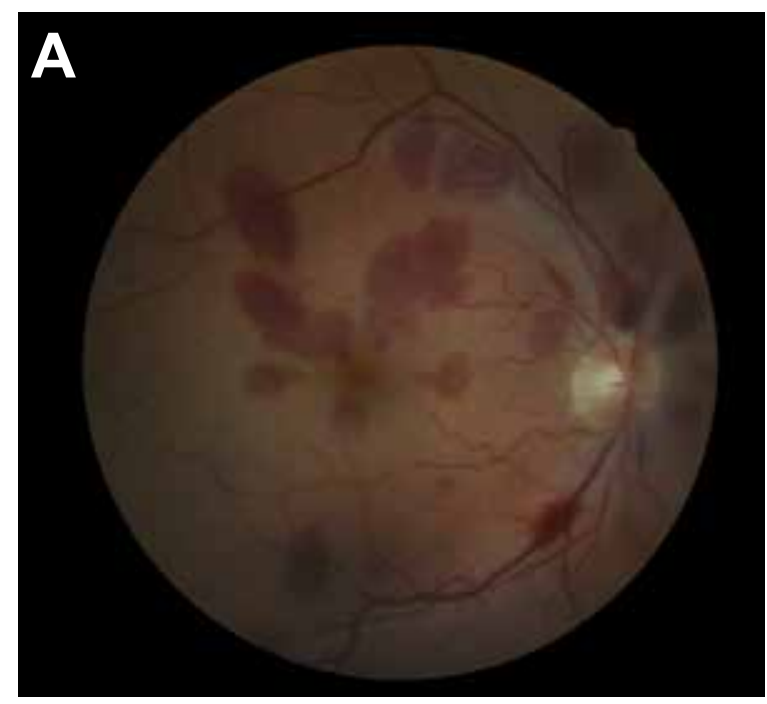

A previous case series on visual impairment following regular epidural fluid injections and epiduroscopy reported 12 cases, $85 \%$ of whom were women (5). The volume of fluid injected into the epidural space varied from $20 \mathrm{~mL}$ to $120 \mathrm{~mL}$. Fifty-eight percent had bilateral hemorrhages; on follow-up almost $80 \%$ had fully recovered (5).

Kushner and Olsen reported 4 cases of retinal hemorrhage after more than 10,000 epidural injections, making it a rare but not insignificant complication (8). The true occurrence of retinal hemorrhage is likely to be higher, because some of the patients may remain asymptomatic or may not attribute their complaints to the procedure.

Potential risk factors for this complication are female sex, obesity, hypertension, coagulopathies, preexisting increase in CSF pressure, and retinal vascular diseases (9).

Performing epiduroscopies should be done with these risks in consideration, and patients with preexisting vision problems or eye diseases are normally excluded from this procedure.

During the procedure, care should be taken that

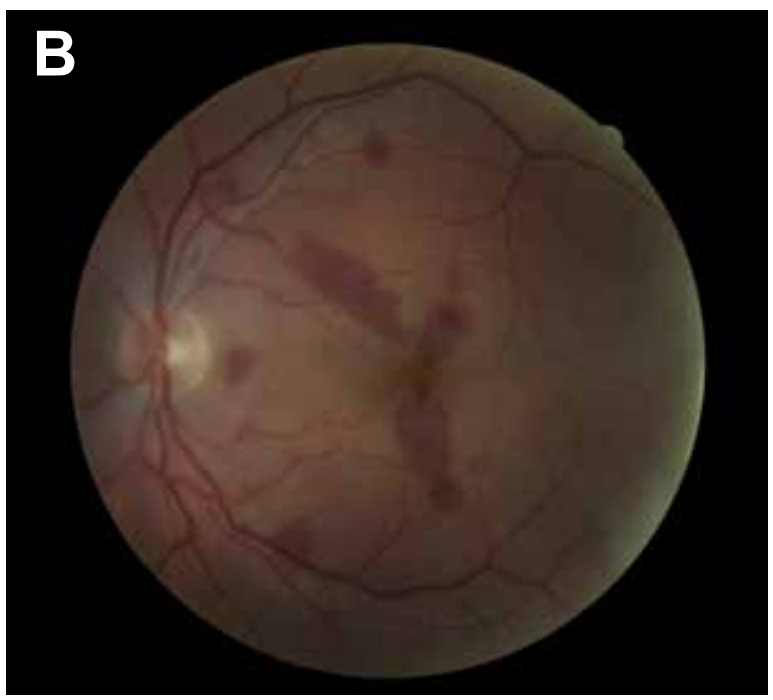

Fig. 1. Fundoscopic photograph of the right (1A) and left (1B) eye showing retinal hemorrhages 
there is no overpressurization of the epidural space, in which case the patient will complain of head and neck pain. More importantly, fluid instillation should be done with a constant pressure; pressure waves caused by intermittent injection of fluid should be avoided.

The patient in this case report received long-term dexamethasone and despite all precautions not to overpressurize the epidural space, the patient did develop retinal hemorrhages.

The immunosuppressive effects of corticosteroids are therapeutic, but they also cause serious side effects. Ocular complications of corticosteroid use, such as cataract glaucoma and retinal emboli, are well known (10). In abdominal surgery, corticosteroid use is associated with an increased risk of complications, with patients receiving a higher dose being at higher risk (11). Chronic corticosteroid use may weaken retinal veins, making them prone to rupture when there is increased pressure, even for a short period of time. Therefore, we hypothesize that longterm use of corticosteroids can be a risk factor for developing retinal hemorrhages after epiduroscopy. As far as we are aware, this is the first description of retinal hemorrhaging after epiduroscopy in a patient using dexamethasone.

\section{CONCLUSIONS}

Chronic use of steroids must be considered a risk factor for developing retinal hemorrhages in patients undergoing epiduroscopy. Considering the consequences of retinal hemorrhaging, we believe chronic steroid use should be a relative contraindication for epiduroscopy.

\section{REFERENCES}

1. Kallewaard JW, Vanelderen P, Richardson J, Van Zundert J, Heavner J, Groen GJ. Epiduroscopy for patients with lumbosacral radicular pain. Pain Pract 2014; 14:365-377.

2. Helm S, 2nd, Racz GB, Gerdesmeyer L, Justiz R, Hayek SM, Kaplan ED, El Terany MA, Knezevic NN. Percutaneous and Endoscopic Adhesiolysis in Managing Low Back and Lower Extremity Pain: A Systematic Review and Meta-analysis. Pain Physician 2016; 19:E245-E282.

3. Ozer A. Minimally Invasive Procedures In Spine Surgery, 2015.

4. Clark CJ, Whitwell J. Intraocular haemorrhage after epidural injection. Br Med J 1961; 2:1612-1613.

5. Gill JB, Heavner JE. Visual impairment following epidural fluid injections and epiduroscopy: A review. Pain Med 2005; 6:367374.

6. Bosscher HA, Heavner JE. Lumbosacral epiduroscopy findings predict treatment outcome. Pain Pract 2014; 14:506-514.
7. Bhatia A, Nelson A, Cohen SP. Breaking Bad (Tissue): Epidural Adhesiolysis and Its Outcomes. Anesth Analg 2017; 124:17551757.

8. Kushner $\mathrm{FH}$, Olson JC. Retinal hemorrhage as a consequence of epidural steroid injection. Arch Ophthalmol 1995; 113:309313.

9. Purdy EP, Ajimal GS. Vision loss after lumbar epidural steroid injection. Anesth Analg 1998; 86:119-122.

10. Carnahan MC, Goldstein DA. Ocular complications of topical, peri-ocular, and systemic corticosteroids. Curr Opin Ophthalmol 2000; 11:478-483.

11. Subramanian V, Saxena S, Kang JY, Pollok RC. Preoperative steroid use and risk of postoperative complications in patients with inflammatory bowel disease undergoing abdominal surgery. Am J Gastroenterol 2008; 103:2373-2381. 